REVIEW ARTICLE

\title{
Confabulation: A Guide for Mental Health Professionals
}

\author{
${ }^{1}$ Concordia University, St. Paul, MN, USA \\ ${ }^{2}$ Pathways Counseling Center, St. Paul, MN, USA \\ ${ }^{3}$ The American Institute for the Advancement of Forensic Studies, St. Paul, MN, USA \\ ${ }^{4}$ University of Houston - Clear Lake, TX, USA
}

Jerrod Brown ${ }^{1,2,3^{*}}$, Deb Huntley ${ }^{1}$, Stephen Morgan ${ }^{1}$, Kimberly D Dodson ${ }^{4}$, and Janina Cich ${ }^{1,3}$

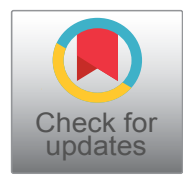

*Corresponding author: Jerrod Brown, Ph.D., MA, MS, MS, MS, Pathways Counseling Center, 1919 University Ave. W. Suite 6 St. Paul MN, 55104, USA, E-mail: jerrod01234brown@live.com

\begin{abstract}
Confabulation is the creation of false memories in the absence of intentions of deception. Individuals who confabulate have no recognition that the information being relayed to others is fabricated. Confabulating individuals are not intentionally being deceptive and sincerely believe the information they are communicating to be genuine and accurate. Confabulation ranges from small distortions of actual memories to creation of bizarre and unusual memories, often with elaborate detail. Although confabulations can occur in non-impaired populations, the aim of this article is to bring into focus the unique problems associated with this phenomenon and its impact on clients involved in the mental health system. The vividness with which clients describe their memories may convince some mental health professionals into believing the memories are real. Even when the client is presented with information that directly conflicts with their version of events, they will persist in believing their memories are wholly accurate. Mental health professionals must be vigilant about its identification to gather accurate information from a client and to provide optimal treatment strategies. Therefore, this article provides information about confabulation, including its characteristics and etiology, links to psychological and neurological disorders, its impact on others, and considerations for mental health professionals.
\end{abstract}

\section{Keywords}

Confabulation, False memory, Mental health, Suggestibility

\section{Introduction}

Confabulation refers to the production or creation of false or erroneous memories without the intent to deceive, sometimes called "honest lying" [1]. Alternatively, confabulation is a falsification of memory by a person who, believes he or she is genuinely communicating truthful memories [2-4]. These false memories may consist of exaggerations of actual events, inserting memories of one event into another time or place, recalling an older memory but believing it took place more recently, filling in gaps in memory, or the creation of a new memory of an event that never occurred [5-7]. While some confabulated memories are easier to identify as false, in other cases, the confabulated memory may be so personal and unique, only a corroborating witness like a family member or close friend may be able to confirm the veracity of the memory. Confabulation also occurs when an individual unintentionally mistakes imagined events as actual memories [8]. Confabulation has been known to occur among clients with brain damage, Fetal Alcohol Spectrum Disorder (FASD), traumatic-brain injury (TBI), and Wernicke-Korsakoff syndrome (WKS). As such, it is imperative for mental health professionals to be familiar with confabulation because of the negative impact it can have on screening, assessment, intake, and treatment planning processes.

\section{Confabulation: Historical Origins and Charac- teristics}

There are several important factors discussed below to assists mental health professionals in becoming more familiar with confabulation. Below is a brief review of the historical origins of confabulation and key characteristics that may assist mental health professionals in identifying it in clients.

\section{Historical origins}

The term confabulation made its first appearance 
in the medical literature in the early 1900s [9]. Sergei Sergeievich Korsakoff, a Russian psychiatrist, noted that alcoholic patients were more likely to display memory deficits referred to as "pseudo-reminiscences, illusions of memory, or falsifications of memory", ultimately called confabulation [10]. Other prominent psychiatrists, such as Emil Kraepelin and Karl Bonhoeffer, began to document cases of confabulation in patients with dementia, senility, and brain trauma $[10,11]$. In such cases, patients can significantly alter important details of memory or generate new memories of events that never took place. However, what is often more problematic is that clients will act on their confabulations, which means their behavior is based on false recall.

\section{Etiological underpinnings}

It is important to note from the outset that in both normal and psychopathologically affected individuals, memory function is constructive. Memories are reconstructions of prior events. As such, within and across the general population, memories are subject to experiential interpositions of the to-be-remembered event and the individual psychological/cognitive state at time of recall. The reconstruction has been flavored by either flawed encoding or intervening cognitions or psychological states so as to render the memory trace non-representative of the actual event. There exists a non-correspondence between the memory of an event and the reality of the event. The literature is replete with examples of memories held by normal, non-pathologically affected individuals, to be firmly and confidently held as true and veridical to the putative prior event, that are simply false. For example, in an experiment by Loftus \& Pickrell [12], parents were recruited to try to convince their children that an early age, they (the child) had been lost in a mall. It was factually confirmed that none of the children were ever lost in a mall. After several sessions of questioning the children about this false experience, $25 \%$ of the child participants reported confidently that they had memories of the non-event, some with impressive (false) detail. In another experiment, Roediger \& McDermott [13] induced false memories in normal subjects in the following way. They gave participants lists of categorically related words to remember, but the list being absent of the critical thematic identifying word, and then asked them to recall the word list. For example, given the words, pillow, bed, sheets, snore, nap, bed, night, pajamas, etc., participants were presented with sleep-related words, but not with the word sleep. In two experiments in their report, participants recalled the non-presented categorical exemplars in $40 \%$ of the trials in experiment 1 , and in $55 \%$ of trials in experiment 2 (the difference in experiments was that experiment 2 used a greater number of semantically related categorical words in the to-be-remembered word list, yet still not including the thematic identifying word). In both experiments 1 and 2 the false memories were recalled with a high degree of confidence as to their veracity.
So, to re-cap, false memories are not unique to pathological populations, they are seen and may be experimentally observed in the normal population. However, given the nature of client-therapist interaction and the consequences of falsely, yet confidently described putative events of the past merits significant note to the informed mental health professional.

Although the etiological underpinnings of psychopathological confabulation are unclear, memory impairments may be linked to basal forebrain damage and self-awareness issues could be contributed to by the frontal lobe [4,14-16]. The roles of these potential contributors can be observed through a common example: When asked what she ate for breakfast this morning, the client recalled consuming oatmeal. This was a false memory because the individual did not have breakfast this morning. Damage to the basal forebrain could have contributed to the client's inability to recall her meal accurately. Further, frontal lobe damage may increase the likelihood that the client believed in the accuracy of her memory, lacking the self-awareness that this was not her experience. Damage to the basal forebrain or frontal lobe (e.g., anterior communicating artery aneurysm) has been known to increase the risk of confabulation [16]. Although confabulation has been observed in individuals with frontal lobe damage, a growing body of research suggests that confabulation may occur in individuals with and without frontal lobe damage [1719]. As such, frontal lobe damage may not be a defining feature of confabulation.

\section{Presentation}

Confabulation can range from small changes or updates to memories of an actual event to the large-scale creation of an event that never took place [20-22]. Confabulations may replace details of a memory with other details that are not true (e.g., eating crab cakes this morning as opposed to the reality of eating eggs, although the person has never eaten crab cakes), placing details of a correct memory into a different occurrence or time frame (e.g., eating oatmeal this morning as opposed to the reality of eating eggs, although oatmeal has sometimes been eaten for breakfast), or simply adding information to the memory that makes sense based on experience of never occurring (e.g., eating grits for breakfast although the person has never had grits before). It is even possible to have incorrect memories for bizarre occurrences that could never have occurred (e.g., "remembering" that the President visited your home on your birthday and brought you breakfast). Regardless, individuals remain confident in these confabulated memories, even when confronted by contradictory evidence [16].

\section{Contributing factors}

According to Gilboa and Verfaillie [23], there are at least three contributing factors to confabulation. First, 
an adequate memory of a specific event may never have been encoded. Second, memories of another event may have altered the memories of the original event, resulting in a memory that is no longer accurate. Third, the individual may possess an unconscious motive to alter the memory.

\section{False memory}

Characterized as a false memory or "honest lying", confabulation can take on several forms [1]. On the one hand, confabulation may be the exaggeration of an existing memory or the coalescence of different existing memories. On the other hand, confabulation can be the outright generation of an entirely new memory of a fictional event. Regardless of the form of confabulation, it is essential to investigate the veracity of memories through collateral informants and other corroborating evidence.

\section{Memory loss}

Confabulation has been hypothesized as a way for individuals to compensate for memory loss. That is, an individual with memory loss may simply confabulate to fill in a gap in their memory [24,25]. However, not all individuals who suffer from memory loss exhibit confabulation. For example, the severity of amnesia is not associated with the presence or frequency of confabulation. Further, confabulation may even occur in the absence of memory-related impairments $[17,19]$.

\section{Lying vs. confabulation}

Confabulation is distinct from lying. By definition, individuals who suffer from confabulation are not aware that the memory is inaccurate and are not intentionally trying to deceive anyone [26]. Mental health professionals may misinterpret confabulation as a malicious attempt to deceive rather than the product of neuropsychological deficits. Whether distorted memories of an actual event or the creation of an imagined event, confabulation is not the same thing as lying. Confabulation also differs from malingering in that the person who confabulates is not doing it for any secondary gain or personal incentive [27].

\section{Spontaneous vs. provoked confabulation}

Current theory suggests that confabulations fall into two sub-classes - provoked and spontaneous [19]. Provoked confabulations are those generated in response to a question. Researchers believe that provoked confabulations are a failure of temporal correspondence to real memories $[25,28,29]$. Spontaneous confabulations are considered a failure of "reality monitoring" -a frontal lobe function [5,30,31]. They are independently generated and are more "fantastic" and "grandiose" in nature and occur without any prompting.

\section{Verbal vs. behavioral}

Confabulation can be manifested in different ways. Verbal confabulation refers to the statement of a false, distorted, or misinterpreted memory whereas behavior confabulation happens when an individual takes physical actions in response to their false memory [10,26,32]. An example of behavioral confabulation would be making a birthday cake for a spouse whose birthday was two months ago. Both types of confabulation still reflect inaccurate memories and may be difficult to detect by mental health professionals given the confidence that is displayed by the individual in the accuracy of the memory.

\section{Memory confidence}

People tend to assume that the accuracy of a memory increases with the reporter's level of confidence in the memory. However, clients may be reporting a confabulated memory with a greatly inflated level of confidence. The confabulation is false, by definition, yet they have confidence in its accuracy [33]. As such, mental health professionals should be careful not to mistake a client's confidence in memory as an indicator of the memory's accuracy. Fiala and Nichols [34], on the other hand, cite studies that show that the individuals who confabulate are initially less confident in its accuracy, often beginning their false recollection with, "I don't know".

\section{Delusions vs. confabulation}

Delusions are false beliefs that usually occur in the context of a psychiatric disorder (e.g., schizophrenia or other psychotic disorder) $[35,36]$. In contrast, confabulations are false memories created to address gaps in memory. These confabulations usually occur in the presence of a neurological condition like dementia or Wernicke-Korsakoff Syndrome (WKS). Although both delusions and confabulations are false beliefs, there are key ways to distinguish them $[37,38]$. If the false belief is related to the recall of memories, the belief is probably confabulation. In contrast, delusions are more likely to influence an individual's behavioral choices across an array of situations. For example, being convinced that a spouse's birthday is this month as opposed to two months ago was noted as an example of confabulation. On the other hand, believing that a deceased spouse continues to exist through brain waves and controls how the other individual acts would be a delusion. Identifying the origins of these false beliefs is an important step for the treatment of these impairments.

\section{Populations Prone to Confabulate}

While confabulations can occur in individuals with no identified deficits or disorders, there are certain psychological and neurological disorders that may increase the incidence of confabulation. Mental health professionals should consider the possibility of confabulation when working with the following populations.

\section{Alzheimer's disease}

Alzheimer's disease is among the most common 
types of dementia. It is a progressive disease that interferes with a variety of cognitive tasks, including memory, thinking, planning, and the apprehension of reality [39]. The diagnosis of Alzheimer's disease must include a decline in memory as well as a decline in at least one area of cognitive functioning such as language comprehension or production, correctly recognizing objects, and planning and carrying out complex tasks [40]. The subjective cognitive decline may be one of the first markers of this disorder [41]. Alzheimer's disease entails neuronal as well as synaptic loss. Additionally, there are positive identifiers that include "plaques" and "tangle" - proteins that interfere with proper neuronal communication. It is currently theorized that the progressive nature of neuronal degeneration comes to affect the frontal lobe, and "reality monitoring" is compromised. It is this degradation of the reality monitoring functioning of the frontal lobe that suggests a causal role in the construction and belief in confabulations [42-45].

\section{Anosognosia}

Anosognosia is a condition in which the afflicted denies having an illness or disorder, which is readily apparent to all others $[46,47]$. The most frequent occurrence is associated with those afflicted by stroke, and subsequently, suffer hemiplegia-the loss or dramatic reduction in the ability to coordinate motor activity on one lateral side of the body (right or left). When asked, for instance, to move the paralyzed arm to touch the nose, the patient may respond that he or she is "tired", or may, in fact, insist that the act was successfully completed, when indeed it was not, which could be considered a form of confabulation.

\section{Anton's syndrome}

Anton's syndrome is a rare disorder. It is a type of anosognosia in which the patient is blind, yet denies being so. The individual maintains with high confidence that they suffer no visual deficits. From a physiological basis, there is absolute blindness, but the patient reports visual perceptions $[48,49]$. If asked to describe the surroundings of the doctor's office, for instance, they will report the typical scene of the typical doctor's office. As in other cases of confabulation, there is no intent to deceive; they have a false belief in their ability to perceive the world visually.

\section{Fetal Alcohol Spectrum Disorder (FASD)}

Fetal Alcohol Spectrum Disorder (FASD) is a life-long persistent neurodevelopmental disorder resulting from prenatal alcohol exposure (PAE). This disorder is characterized by a diverse array of cognitive (e.g., short- and long-term memory, intelligence, and attention), social (e.g., social pressure, social cues, and communication skills), and adaptive (e.g., decision-making and problem-solving abilities) deficits [50-54]. As a result of the array of deficits associated with FASD, individuals impacted by this disorder may be at an increased risk to confabulate compared to their non-FASD counterparts. Confabulation of new memories from real and fictional experiences is a common issue in FASD. A combination of the cognitive (e.g., executive deficits), social (e.g., suggestibility, eagerness to please others), and adaptive deficits of FASD likely contribute to the manifestation of confabulation. In response to memory gaps, individuals with FASD may confabulate to render a complete story [8].

\section{PTSD}

Post-traumatic stress disorder (PTSD) is noted in the Diagnostic and Statistical Manual of Mental Disorders [55] as a disorder that results from a traumatic experience and is characterized by symptoms such as intrusion, avoidance, negative affect and/or thoughts, and increased arousal. Individuals who have increased anxiety and trauma may be more prone toward confabulation. The stress incurred during the trauma may interfere with memory encoding and retrieval. Interviewers and therapists should maintain a calm demeanor and not engage in confrontational questioning.

\section{Schizophrenia}

Schizophrenia, commonly viewed as a thought disorder, is noted in the Diagnostic and Statistical Manual of Mental Disorders, $5^{\text {th }}$ edition (DSM-5) as a brain disorder that affects how individuals think (e.g., delusions), feel (e.g., flat or inappropriate affect), and perceive (e.g., hallucinations) [55]. The presence of schizophrenia may increase the risk for confabulation [56]. In a study by Simpson and Done [36], patients diagnosed with delusional schizophrenia demonstrated confabulation more than both patients without delusions and control subjects without mental health issues. Further, patients diagnosed with delusional schizophrenia exhibited memory errors during the recall of stories but not word lists. This is in contrast to patients with traumatic brain injuries, where errors are common during both the recall of stories and word lists. As such, confabulation may be related to the verbal comprehension impairments and organizational deficits of schizophrenia. Nonetheless, confabulation must be carefully distinguished from disordered thought, as both have the potential to be consistent across time [57].

\section{Traumatic-brain injury}

"A traumatic brain injury (TBI) occurs when an external force alters brain functioning or results in some other form of brain injury" [58]. TBIs can be characterized by deficits in neurological processes and memory along with changes in mental states and consciousness. Confabulation may be a symptom of traumatic brain injury (TBI), a brain dysfunction that results from a blow to the head $[3,59,60]$. The diagnosis of TBI may be complicated by confabulation [61]. Specifically, the client may simply recall inaccurate information during the assessment process that decreases the likelihood of accurate diag- 
nosis. Asking a client if they have suffered brain injury or been diagnosed with a TBI may not prove helpful to the practitioner. Therefore, consulting with close family and friends may yield a more accurate picture of the client's medical history [61].

\section{Wernicke-Korsakoff Syndrome (WKS)}

Wernicke-Korsakoff Syndrome (WKS) is a neurological condition caused by a vitamin B1 (thiamine deficiency) and is frequently seen in individuals who excessively consume alcohol in conjunction with poor nutritional diets $[62,63]$. Wernicke-Korsakoff Syndrome (WKS) is composed of two related conditions: Wernicke's encephalopathy (WE) and Korsakoff Syndrome (KS). WKS is characterized by cognitive deficits including impairments in executive function and memory. As a result, the presence of WKS often increases the likelihood of confabulation [24, 64-66].

\section{Consequences}

Many consequences can result from confabulation, some of them more serious than others. For example, a person who confabulates may upset and frustrate loved ones, but unintentionally lying to a law enforcement officer or court official could result in arrest and incarceration. Mental health professionals should be aware of these consequences and provide care that is more comprehensive and to help clients avoid these negative outcomes.

\section{Counseling ramifications}

In addition to family members and friends, confabulation can have a profound impact on mental health professionals. Treating these challenging clients is complicated by the fact that mental health professionals cannot rely on the information provided by their clients, which can result in tedious, repetitive, and frustrating interactions [67]. To minimize the magnitude of frustration, mental health professionals should remind themselves that confabulation may be the result of neurological impairment that is beyond the control of the client. It is helpful to corroborate the information by asking family members or using archival data [68].

\section{Family impact}

A systemic approach to addressing confabulation involves consideration of the impact that confabulation has on family members. As can be expected, confabulation often results in family members feeling sad, fearful, frustrated, or angry. Confabulation also affects the trust between family members [61]. To improve the situation, mental health professionals should assure family members that such emotional responses are not uncommon when dealing with a loved one exhibiting confabulation. Further, mental health professionals should make sure that the family recognizes that confabulation is not purposeful and that family support remains important in any intervention. Family members can gently provide evidence or information to help the individual correct the erroneous memory but should not engage in arguing or confrontation $[67,68]$.

\section{Legal considerations}

Because many legal processes strongly rely on the memory of a suspect, defendant, or witness, confabulation can have significant consequences in this arena. If present, confabulation may cast doubt on confessions and eyewitness testimony $[69,70]$. In addition, criminal justice practitioners may perceive a person's recollections to be deceptive and result in increased scrutiny regarding the veracity of the person's recall and/or criminal charges. For example, lying under oath could result in the individual being charged with perjury. Mental health professionals who perform services within the criminal justice system are strongly advised to be familiar with confabulation.

\section{Suggestibility}

Individuals who suffer from confabulation may also be prone to suggestibility $[71,72]$. Specifically, these individuals may be likely to adopt the statements or views of others when prompted by repeated questioning and negative feedback. This is a particular concern during police interrogations and questioning by attorneys during testimony. This can also create challenges during the initial assessment and intake process for mental health treatment. Rather than ask closed ended or no/yes questions, interviewers should ask open-ended questions.

\section{Screening and Assessment}

Confabulation can complicate the screening and assessment process. As previously noted, there are several factors related to confabulation, including a history of brain injury, neurological deficits, false stories that may be detailed or strange, or conviction or confidence in the false memory. Unfortunately, these factors may be indicative of several different mental health disorders. Below we offer some suggestions to improve screening and assessment of confabulation in clinical settings.

\section{Recognition}

Despite appearances of confidence and accuracy, individuals may confabulate entirely false memories of details or events $[1,17]$. As such, the recognition of confabulation remains incredibly difficult for mental health professionals. In such cases, a referral for a neurological evaluation maybe warranted. While certain neurological conditions increase the risk for confabulation, not all cases of confabulation are caused by neurological issues $[67,68]$.

\section{Identification}

There is a lack of consensus on the causal origins of confabulation and, subsequently, the definition of confabulation $[30,37]$. This situation is exacerbated by the 
fact that confabulation co-occurs with several different disorders and even in individuals with no neurological or mental health issues. As a result, mental health professionals often have difficulty recognizing and discerning when confabulation is taking place $[67,68]$. The seriousness of these issues emphasizes why wider awareness of confabulation is essential in the mental health field.

\section{Malingering vs. confabulation}

Malingering is an effortful attempt to deceive whereas confabulation is not purposefully deceptive $[73,74]$. In other words, individuals who are malingering recognize that they are not honest whereas individuals who confabulate believe that they are being honest. As such, as part of a comprehensive screening and assessment process, mental health professionals should become familiar with the similarities and differences between malingering and confabulation.

\section{Missed and misdiagnosis}

Clients who confabulate may over- or under-endorse mental health-related symptoms. As such, confabulation can lead to missed and misdiagnosis. This is especially the case when mental health professionals lack the appropriate awareness of this complex topic. When confabulation is suspected, it is imperative for mental health professionals to fact check, clarify, and verify statements provided by clients who confabulate. This should also include reviewing multiple data sources to confirm accounts provided by clients with a history of confabulation $[67,68,73,75]$.

\section{Other considerations}

The presence of memory deficits increases the risk for confabulation [76-78]. Such deficits could relate to autobiographical memories or source monitoring $[4,79]$. Further, individuals with active fantasy lives, gullibility, and overactive imaginations could be prone to confabulation. Similarly, confabulation can even be influenced by something as common as everyday interactions with others or social media. These are all areas that should be considered during the screening and assessment process.

\section{Knowledge deficit}

All confabulations share a central theme - a knowledge deficit. The deficit can be related to the corporeal body, as seen in anosognosia. The deficit can be of knowledge of reality, as seen in "spontaneous" confabulations, and in Alzheimer's disease. The deficit can be of knowledge of accurate temporal mapping to relatively reasonable "memories", as seen in provoked confabulations. Finally, the deficit can concern knowledge of our own perceptions, as seen in Anton's syndrome.

\section{Strategies and Solutions}

Confabulation frequently co-occurs with several disorders and presents in different ways, but all have the potential to impact accurate diagnosis and successful treatment. Mental health professionals should seek out education and training opportunities to help them identify instances of confabulation in their clients. In addition to correctly identifying confabulation, there are some things that mental health professionals can do when confabulation is suspected to treat the patient.

\section{Treatment}

In some cases, confabulation dissipates without treatment, whereas other clients may need treatment to help them recognize and reduce their tendency to confabulate. Effective interventions for confabulation may require that mental health professionals establish and maintain working relationships with other professionals and providers who possess experience in dealing with confabulation in mental health settings $[67,68,73,75]$.

\section{Corroborate information}

When mental health professionals work with a client who may be confabulating, it is vital that the professional tries to corroborate any information self-reported by the client. This could take the form of talking to collateral informants like friends and family members or finding corroborating evidence such as medical or criminal justice records $[67,68,73,75]$. The incorporation of these methods has the potential to improve assessment and, in turn, treatment outcomes. The process of corroboration is particularly important in situations where memories can have a profound impact.

\section{Establishing the therapeutic relationship}

When working with clients who may be confabulating, mental health professionals must keep in mind, that confabulation is not purposeful or mischievous in intent. To establish an effective therapeutic relationship, mental health professionals may consider employing a relaxed pace, open-ended questions, and easy-to-understand language. Methods that may be beneficial include using memory diaries and introducing the client to patient self-monitoring and other ways to monitor memories $[80,81]$. Finally, the confabulations themselves may provide glimpses into the client's triggers for confabulation and other treatment needs.

\section{Support system}

If an individual suffers from confabulation, the presence of a strong support system is imperative to ensure the accurate communication of personal and health-related information. The reliance on such a group of collateral informants has the potential to maximize the accuracy of information when making important life-altering decisions $[67,68,73,75]$. The mental health professional, with permission from the client, should educate the family about confabulation and outline what can be expected and how to address confabulations. This knowledge gives the family an understanding and keeps them engaged with the individual. 


\section{Conclusion}

Confabulation can have varied effects across all mental health-related settings. For example, confabulation can bring forth a number of consequences in mental health treatment settings. Foremost, accurately identifying confabulation can be difficult for mental health professionals. When confabulation goes unrecognized, inaccurate information can contribute to misdiagnoses and less than optimal intervention and treatment strategies. To protect against the harmful effects of confabulation, mental health professionals need to incorporate corroborative information including collateral informants (e.g., family members) and sources (e.g., medical and criminal justice records), seek out additional education and training opportunities, and consult with experts when confabulation is suspected or confirmed.

\section{References}

1. Moscovitch M (1989) Confabulation and the frontal system: Strategic versus associative retrieval in neuropsychological theories of memory. In: HL Roediger, FIM Craik, Varieties of memory and consciousness: Essays in honour of Endel Tulving. Lawrence Erlbaum Associates, Hillsdale, New Jersey, United States, 133-160.

2. Ciaramelli E, Ghetti S (2007) What are confabulators' memories made of? A study of subjective and objective measures of recollection in confabulation. Neuropsychologia 45: 1489-1500.

3. Gilboa A, Moscovitch M (2002) The cognitive neuroscience of confabulation: A review and a model. In: Baddeley AD, Kopelman MD, Wilson BA, The Handbook of Memory Disorders. John Wiley \& Sons Ltd.

4. Johnson MK, O'Connor M, Cantor J (1997) Confabulation, memory deficits, and frontal dysfunction. Brain Cogn 34: 189-206.

5. Johnson MK (1991) Reality monitoring: Evidence from confabulation in organic brain disease patients. In: GP Prigatano, DL Schacter, Awareness of deficit after brain injury Oxford. Oxford University Press, 176-197.

6. Kopelman MD, Guinan EM, Lewis PDR (1995) Delusional memory, confabulation and frontal lobe dysfunction: A case study in de clérambault's syndrome. Neurocase 1: 71-77.

7. Nedjam Z, Devouche E, Dalla Barba G (2004) Confabulation, but not executive dysfunction discriminate $A D$ from frontotemporal dementia. Eur J Neurol 11: 728-733.

8. Brown J (2017) Fetal alcohol spectrum disorder and confabulation: A clinical, forensic, and judicial dilemma. The Journal of Special Populations 1: 1-11.

9. Berrios GE (1998) Confabulations: A conceptual history. Journal of the History of the Neurosciences 7: 225-241.

10. Schnider A (2008) The confabulating mind: How the brain creates reality. Oxford University Press, New York.

11. Ebert A, Bär KJ (2010) Emil Kraepelin: A pioneer of scientific understanding of psychiatry and psychopharmacology. Indian J Psychiatry 52: 191-192.

12. Loftus E, Pickrell J (1995) The formation of false memories. Psychiatric Annals 25: 720-725.

13. Roediger H, Mc Dermott K (1995) Creating false memories: Remembering words not presented in lists. Journal of Ex- perimental Psychology: Learning, Memory, and Cognition 21: 803-814.

14. Kapur N, Coughlan AK (1980) Confabulation and frontal lobe dysfunction. J Neurol Neurosurg Psychiatry 43: 461463.

15. Moscovitch M, Melo B (1997) Strategic retrieval and the frontal lobes: Evidence from confabulation and amnesia. Neuropsychologia 35: 1017-1034.

16. Turner MS, Cipolotti L, Yousry TA, Shallice T (2008) Confabulation: Damage to a specific inferior medial prefrontal system. Cortex 44: 637-648.

17. Burgess PW, Shallice T (1996) Confabulation and the control of recollection. Memory 4: 359-411.

18. Dalla Barba G (1993) Confabulation: Knowledge and recollective experience. Cognitive Neuropsychology 10: 1-20.

19. Kopelman MD (1987) Two types of confabulation. J Neurol Neurosurg Psychiatry 50: 1482-1487.

20. Anastasi JS (2006) Understanding confabulation: A multidisciplinary approach. Applied Cognitive Psychology 20: 275-278.

21. Macleod E, Gross J, Hayne H (2016) Drawing conclusions: The effect of instructions on children's confabulation and fantasy errors. Memory 24: 21-31.

22. Shingaki H, Park P, Ueda K, Murai T, Tsukiura T (2016) Disturbance of time orientation, attention, and verbal memory in amnesic patients with confabulation. J Clin Exp Neuropsychol 38: 171-182.

23. Gilboa A, Verfaellie M (2010) Telling it like it isn't: The cognitive neuroscience of confabulation. $J$ Int Neuropsychol Soc 16: 961-966.

24. Bonhoeffer K (1904) Der Korsakowsche Symptomenkoplex in seinen Beziehungenzu den verschiedenen Krankheitsformen. Allgemeine Zeitung Psychiatrie 61: 744-752.

25. Dalla Barba G, Boisse MF (2010) Temporal consciousness and confabulation: Is medial temporal lobe "temporal"? Cognitive Neuropsychiatry 15: 95-117.

26. Moscovitch M (1995) Confabulation. In: DL Schacter, JT Coyle, GD Fischbach, MM Mesulam, LE Sullivan, Memory distortion: How minds, brains, and societies reconstruct the past. Harvard University Press, Cambridge, 226-251.

27. Brown J, Long-McGi J, Oberoi P, Wartnik A, Wresh J, et al. (2014) Confabulation: Connections between brain damage, memory and testimony. The Journal of Law Enforcement 3: 1-11.

28. Budson AE (2009) Understanding memory dysfunction. Neurologist 15: 71-79.

29. Dickerson BC, Eichenbaum H (2010) The episodic memory system: Neurocircuitry and disorders. Neuropsychopharmacology 35: 86-104.

30. Fotopoulou A, Conwa MA, Solm M (2007) Confabulation: Motivated reality monitoring. Neuropsychologia 45: 21802190.

31. Schnider A (2001) Spontaneous confabulation, reality monitoring, and the limbic system-a review. Brain Res Brain Res Rev 36: 150-160.

32. Rensen YC, Oosterman JM, van Damme JE, Griekspoor SI, Wester AJ, et al. (2015) Assessment of confabulation in patients with alcohol related cognitive disorders: The Nijmegen-Venray Confabulation List (NVCL-20). Clin Neuropsychol 29: 804-823. 
33. Zaragoza MS, Payment KE, Ackil JK, Drivdahl SB, Beck $M(2001)$ Interviewing witnesses: Forced confabulation and confirmatory feedback increase false memories. Psychol Sci 12: 473-477.

34. Fiala B, Nichols S (2009) Confabulation, confidence, and introspection. Behavioral and Brain Sciences 32: 144-145.

35. Fotopoulou A (2010) The affective neuropsychology of confabulation and delusion. Cogn Neuropsychiatry 15: 38-63.

36. Simpson J, Done DJ (2002) Elasticity and confabulation in schizophrenic delusions. Psychol Med 32: 451-458.

37. Kopelman MD (2010) Varieties of confabulation and delusion. Cogn Neuropsychiatry 15: 14-37.

38. Turner M, Coltheart M (2010) Confabulation and delusion: A common monitoring framework. Cogn Neuropsychiatry 15: 346-376.

39. Wells CE, Whitehouse PJ (1996) Cortical dementia. In: BS Fogel, RB Schiffer, SM Rao, Neuropsychiatry. Williams and Wilkins, Baltimore, Maryland.

40. Alzheimer's Association (2013) Alzheimer's disease facts and figures. Alzheimer's \& Dementia 9: 208-245.

41. Jessen F, Amariglio RE, Van Boxtel M, Breteler M, Ceccaldi $\mathrm{M}$, et al. (2014) A conceptual framework for research on subjective cognitive decline in preclinical Alzheimer's disease. Alzheimers Dement 10: 844-852.

42. Balota DA, Cortese MJ, Duchek JM, Adams D, Roediger III HL, et al. (1999) Veridical and false memories in healthy older adults and in dementia of the Alzheimer's type. Cognitive Neuropsychology 16: 361-384.

43. Budson AE, Daffner KR, Desikan R, Schacter DL (2000) When false recognition is unopposed by true recognition Gist-based memory distortion in Alzheimer's disease. Neuropsychology 14: 277-287.

44. Kern RS, Van Gorp WG, Cummings JL, Brown WS, Osato $S$, et al. (1992) Confabulation in Alzheimer's disease. Brain Cogn 19: 172-182.

45. Nedjam Z, Dalla Barba G, Pillon B (2000) Confabulation in a patient with fronto - temporal dementia and a patient with Alzheimer's disease. Cortex 36: 561-577.

46. Perrotin A, Desgranges B, Landeau B, Mezenge F, La Joie, et al. (2015) Anosognosia in Alzheimer disease: Disconnection between memory and self related brain networks. Ann Neurol 78: 477-486.

47. Vannini $P$, Dagley AS, McLaren D, Schultz AP, Rentz DM, et al. (2015) Neurobiological correlates of anosognosia in mild cognitive impairment: A multi-modal investigation using FDG-PET, PiB-PET, and volumetric MRI. Alzheimer's \& Dementia: The Journal of the Alzheimer's Association 11: 60.

48. Goldenberg G, Mu W, Nowak A (1995) Imagery without perception-a case study of anosognosia for cortical blindness. Neuropsychologia 33: 1373-1382.

49. Maddula M, Lutton S, Keegan B (2009) Anton's syndrome due to cerebrovascular disease: A case report. J Med Case Rep 3: 9028.

50. Burd L, Selfridge R, Klug M, Bakko S (2004) Fetal alcohol syndrome in the United States corrections system. Addict Biol 9: 169-176.

51. Fast Dk, Conry J (2006) The Challenge of Fetal Alcohol Syndrome in the Criminal Legal System. Addict Biol 9: 161166.
52. Fast DK, Conry J, Loock CA (1999) Identifying fetal alcohol syndrome among youth in the criminal justice system. J Dev Behav Pediatr 20: 370-372.

53. Guerri C, Bazinet A, Riley EP (2009) Fetal Alcohol Spectrum Disorders and Alterations in Brain and Behavior. Alcohol Alcohol 44: 108-114.

54. Jirikowic T, Kartin D, Olson HC (2008) Children with fetal alcohol spectrum disorders: a descriptive profile of adaptive function. Can J Occup Ther 75: 238-248.

55. American Psychiatric Association (2013) Diagnostic and statistical manual of mental disorders. ( $5^{\text {th }}$ edn), American Psychiatric Publishing, Arlington, VA.

56. Nathaniel-James DA, Frith CD (1996) Confabulation in schizophrenia: Evidence of a newform. Psychol Med 26: 391-399.

57. Asp EW, Tranel D (2013) False Tagging Theory: Toward a unitary account of prefrontal cortex function. In: Stuss DT, Knight RT, Principles of Frontal Lobe Function. ( $2^{\text {nd }}$ edn), Oxford University Press, New York, 383-416.

58. Brown J, Louie J (2016) Traumatic-Brain Injury (TBI) and the criminal justice system: An introduction to a complex topic. Forensic Scholars Today 2: 1-4.

59. Demery JA, Hanlon RE, Bauer RM (2001) Profound amnesia and confabulation following traumatic brain injury. Neurocase $7:$ 295-302.

60. Johnson MK, Hayes SM, DEsposito M, Raye C (2000) Confabulation. In: F Boller, J Grafman, Handbook of neuropsychology: Memory and its disorders. (2nd edn), Elsevier Science, Amsterdam, The Netherlands, 383-407.

61. Brown J, Huntley D, Asp E, Fabrizio K, Wiley C, et al. (2016) Traumatic Brain Injury and Confabulation: An Introduction for Criminal Justice and Legal Professionals. The Journal of Special Populations 1: 1-13.

62. Isenberg-Grzeda E, Kutner HE, Nicolson SE (2012) Wernicke-Korsakoff-syndrome: Under-recognized and under-treated. Psychosomatics 53: 507-516.

63. Scalzo SJ, Bowden SC, Ambrose ML, Whelan G, Cook MJ (2015) Wernicke Korsakoff syndrome not related to alcohol use: A systematic review. J Neurol Neurosurg Psychiatry 86: 1362-1368.

64. Kopelman MD, Ng N, Van Den Brouke O (1997) Confabulation extending across episodic, personal, and general semanticmemory. Cognitive Neuropsychology 14: 683-712.

65. Mercer B, Wapner W, Gardner H, Benson DF (1977) A study of confabulation. Arch Neurol 34: 429-433.

66. Talland GA (1961) Confabulation in the Wernicke-Korsakoff syndrome. J Nerv Ment Dis 132: 361-381.

67. Huntley D, Brown J, Wiley CR (2016) Confabulation and mental health: A beginner's guide. Behavioral Health 4: 1-9.

68. Huntley D, Brown J (2016) Understanding Confabulation: An Introduction for Criminal Justice and Mental Health Professionals. Forensic Scholars Today 1: 1-4.

69. Clare ICH, Gudjonsson GH (1995) The Vulnerability of Suspects with Intellectual Disabilities During Police Interviews: A Review and Experimental Study of Decision Making. Mental Handicap 8: 110-128.

70. Smith P, Gudjonsson GH (1995) Confabulation among forensic inpatients and its relationship with memory, suggestibility, compliance, anxiety, and self-esteem. Personality and Individual Differences 19: 517-523. 
71. Gudjonsson GH (2003) The psychology of interrogations and confessions: A handbook. Wiley, Chichester, UK.

72. Gudjonsson GH, Clare IC (1995) The relationship between confabulation and intellectual ability, memory, interrogative suggestibility and acquiescence. Personality and Individual Differences 19: 333-338.

73. Brown J, Hesse M, Rosenbloom M, Harris B, Weaver J, et al. (2015) Confabulation in correctional settings: An exploratory review. The Journal of Law Enforcement 4: 1-8.

74. Vitacco MJ, Jackson RL, Rogers R, Neumann CS, Miller HA (2008) Detection strategies for malingering with the miller forensic assessment of symptoms test a confirmatory factor analysis of its underlying dimensions. Assessment 15: 97-103.

75. Mertz C, Brown J (2015) Confabulation: An introduction for psychologists. Forensic Scholars Today 1: 1-2.

76. De Luca J (2000) A cognitive perspective on confabulation.
Neuropsychoanalysis 2: 119-132.

77. Feinberg TE, Roane DM (1997) Anosognosia. In: JF Feinberg, MJ Farah, Behavioural Neurology and Neuropsychology. ( $2^{\text {nd }}$ edn), McGraw Hill, New York, 324-362.

78. Hirstein W (2005) Brain fiction: Self-deception and the riddle of confabulation. MIT Press, Cambridge, MA, USA.

79. Johnson MK, Hashtroudi S, Lindsay DS (1993) Source monitoring. Psychol Bull 114: 3-28.

80. Alderman N, Fry RK, Youngson HA (1995) Improvement of self-monitoring skills, reduction of behaviour disturbance and the dysexecutive syndrome: Comparison of response cost and a new programme of self-monitoring training. Neuropsychological Rehabilitation 5: 193-221.

81. Dayus B, Vanden Broek MD (2000) Treatment of stable delusional confabulations using self-monitoring training. Neuropsychological Rehabilitation 10: 415-427. 